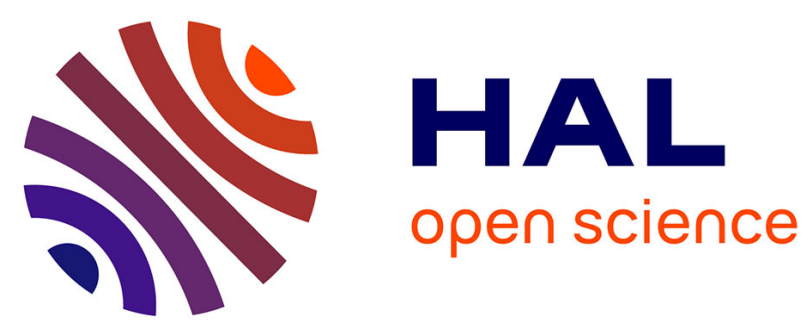

\title{
ESR Investigation of Chemical Exchange in Geminally Diphosphorylated Linear Nitroxide Radicals
}

Antal Rockenbauer, Gilles Olive, Xavier Rozanska, Alain Jacques, Didier

Gigmes, François Le Moigne, Daniel Peeters, Anton German, Paul Tordo

\section{- To cite this version:}

Antal Rockenbauer, Gilles Olive, Xavier Rozanska, Alain Jacques, Didier Gigmes, et al.. ESR Investigation of Chemical Exchange in Geminally Diphosphorylated Linear Nitroxide Radicals. Journal of Physical Chemistry A, 2004, 108 (25), pp.5484-5489. hal-00662572

HAL Id: hal-00662572

https://hal.science/hal-00662572

Submitted on 24 Jan 2012

HAL is a multi-disciplinary open access archive for the deposit and dissemination of scientific research documents, whether they are published or not. The documents may come from teaching and research institutions in France or abroad, or from public or private research centers.
L'archive ouverte pluridisciplinaire HAL, est destinée au dépôt et à la diffusion de documents scientifiques de niveau recherche, publiés ou non, émanant des établissements d'enseignement et de recherche français ou étrangers, des laboratoires publics ou privés. 


\section{ESR Investigation of Chemical Exchange in Geminally Diphosphorylated Linear Nitroxide Radicals}

Antal Rockenbauer ${ }^{\natural}$, Gilles Olive ${ }^{\dagger *}$, Xavier Rozanska, Alain Jacques, Didier Gigmes ${ }^{\S}$, François Le Moigne ${ }^{\S}$ Daniel Peeters, Anton German ${ }^{\dagger}$, Paul Tordo ${ }^{\S}$

${ }^{\star}$ Institute of Chemistry, Chemical Research Center, Budapest, P. O. Box 17, H-1525 Hungary. Department of Polymer Chemistry and Coatings Technology, Eindhoven University of Technology, P.O. Box 513, 5600 MB Eindhoven, The Netherlands. ${ }^{\ddagger}$ Schuit Institute of Catalysis, Laboratory of Inorganic Chemistry and Catalysis, Eindhoven University of Technology, P.O. Box 513, 5600 MB Eindhoven, The Netherlands.`Unité CSTR, Université catholique de Louvain, Bâtiment Lavoisier, Place Louis Pasteur, 1, B-1348 Louvain-la-Neuve, Belgium. ${ }^{\S}$ Laboratoire Structure et Réactivité des Espèces Paramagnétiques, CNRS UMR 6517, Chimie, Biologie et Radicaux Libres, Universités d'AixMarseille I et III, Centre de St Jérôme, Service 521, Avenue Escadrille Normandie-Niemen, 13397 Marseille Cedex 20, France.

Gilles.olive@excite.com

RECEIVED DATE (to be automatically inserted after your manuscript is accepted if required according to the journal that you are submitting your paper to)

ESR Investigation of Diphosphorylated Linear Nitroxide

* to whom correspondence should be addressed. Current address: Rue Maison d'Orbais, 23/14, B-5032 Corroy-le-Château, Belgium. 
ABSTRACT For geminally diphosphorylated linear nitroxide radicals, a marked line width alternation (LWA) can be observed. The LWA appears irrespectively whether the investigated radicals $\mathrm{R}^{\prime \prime} \mathrm{N}\left(\mathrm{O}^{\circ}\right) \mathrm{C}\left(\mathrm{P}(\mathrm{O})(\mathrm{OEt})_{2}\right)_{2} \mathrm{R}^{\prime}$ contain any chiral group or not. If $\mathrm{R}^{\prime \prime}=$ tertio-butyl and $\mathrm{R}^{\prime}=\mathrm{H}$, the small $\beta$ hydrogen coupling indicates a completely blocked rotation around the $\mathrm{N}-\mathrm{C} \sigma$-bond and the LWA can be assigned to a chemical exchange between conformations in which the phosphoryl groups have a symmetric and a non-symmetric geometry. The conformational change is accompanied with the deformation of the $\mathrm{CP}_{2}$ bonding angle and the rate of exchange is slowed down in pentane for which the solvent molecules can be trapped by the chelating phosphoryl groups. If R"=benzyl, both the proton hyperfine lines of the $\mathrm{CH}_{2}$ group and the phosphorus lines of the $\mathrm{CP}_{2}$ group produce a LWA ; two coalescences can be observed. In the case of a chiral R" group (R"=secondary butyl, R'=methyl) a tentative four-site model can explain the highly complex LWA.

\section{Introduction}

In our earlier study ${ }^{1}$, we found that the exchange phenomenon is rather complex for the geminally diphosphorylated cyclic nitroxides with pyrrolidine rings. In this case, the concerted motion of the C-P rotation of phosphoryl groups as well as the ring interconversion yield complex exchange processes. Due to the actual 'four-site' character, two distinct coalescences can be observed.

In this paper, we are to extend our studies to the rotation of the geminal $\mathrm{CP}_{2}$ group in linear radicals. The investigated radicals structure is $\mathrm{R}^{\prime \prime} \mathrm{N}\left(\mathrm{O}^{\circ}\right) \mathrm{C}\left(\mathrm{P}(\mathrm{O})(\mathrm{OEt})_{2}\right)_{2} \mathrm{R}^{\prime}$ where $\mathrm{R}^{\prime}$ and $\mathrm{R}^{\prime \prime}$ represent various alkyl groups (see Table 1 and Scheme 1). Although a line width alternation (LWA) in the ESR spectra of the

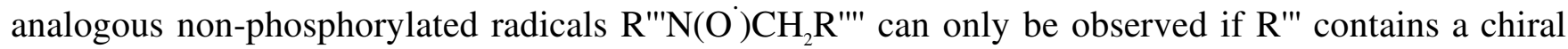
carbon atom ${ }^{2}$, the geminal diphosphorylation yields to LWA even for radicals without any chiral center. We will show this phenomenon in radicals with non-chiral $\mathrm{R}$ ' $(\mathrm{H}$, methyl or ethyl group) when $\mathrm{R}^{\prime \prime}$ is either non-chiral (tertio-butyl, benzyl) or chiral (sec-butyl). We will analyze the impact of the 
chirality onto the LWA. Since the crowded motion of geminal phosphoryl groups is also influenced by the molecular interaction with solvents, we will also investigate this effect on the LWA. ${ }^{3}$

Table 1: Structure of the studied compounds

\begin{tabular}{cccc}
\hline Compounds & $\mathbf{1}$ & $\mathbf{2}$ & $\mathbf{3}$ \\
\hline $\mathrm{R}^{\prime}$ & $\mathrm{H}$ & $\mathrm{Et}$ & $\mathrm{Me}$ \\
$\mathrm{R}^{\prime \prime}$ & $t \mathrm{Bu}$ & $\mathrm{Bn}$ & $s \mathrm{Bu}$
\end{tabular}

Scheme 1: Structure of the studied compounds

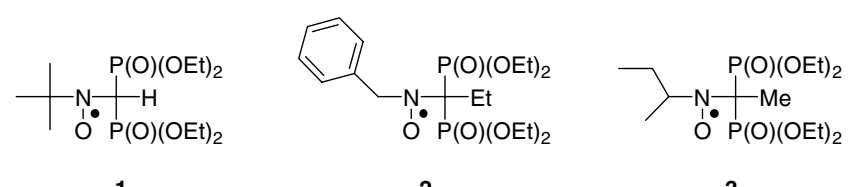

Nitroxides 1, $\mathbf{2}$ and $\mathbf{3}$ were generated as shown in Scheme 2. The synthesis and full characterization of $\mathbf{4}$ and $\mathbf{5}$ have already been described elsewhere. ${ }^{4} \mathbf{6}$ was prepared as depicted in Scheme $\mathbf{3}$ using the previously reported one-step diphosphorylation ${ }^{5}$ and its full characterization will be published in a forthcoming paper. The ESR spectrum of $\mathbf{1}$ has already been reported by Il'yasov ${ }^{6}$ and Pedulli. ${ }^{7}$ It has to be mentioned that we succeeded in synthesizing the amine with R"=tertio-butyl and R'=methyl but failed to obtain the corresponding nitroxide.

Scheme 2: Synthesis of nitroxides 1-3

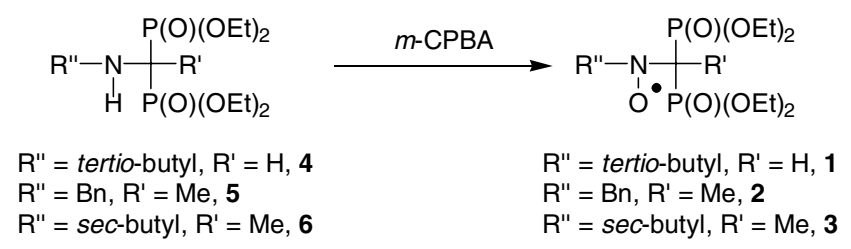


Scheme 3: Synthesis of 6

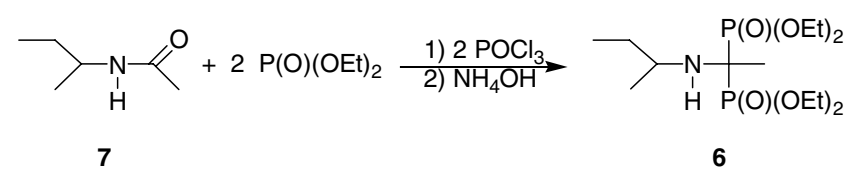

All ESR spectra were simulated using the previously described method and a homemade software. ${ }^{8}$

\section{Experimental Section}

ESR measurements were performed on a Bruker ESP 300E spectrometer equipped with a X-band resonator $(9.41 \mathrm{GHz})$. All ESR spectra were recorded at $100 \mathrm{kHz}$ magnetic field modulation. Solvents were purchased from Biosolve. $m$-CPBA ( $m$-chloroperbenzoic acid) (70-75\%) was used as purchased from Acros. 7 was prepared by using the procedure of Sagar. ${ }^{9}$

ESR Study of Nitroxides 1, 2 and 3. Compounds 4, 5 or $6(0.03 \mathrm{mmol})$ were dissolved in $100 \mu \mathrm{l}$ of solvent. $m$-CPBA $(7.2 \mathrm{mg}, 0.03 \mathrm{mmol})$ was added and the spectrum was recorded immediately after helium bubbling.

\section{Results and Discussion}

\section{Analysis of Radical 1}

The proton coupling provides some additional structural information and the lack of a chiral center makes the phenomenon simpler. The ESR spectra dependence on temperature shows a marked solvent effect: whereas the coalescence region is only reached at $173 \mathrm{~K}$ in dichloromethane, the rate of exchange in $n$-pentane is slower by an order of magnitude. Below $233 \mathrm{~K}$, a superimposed character of lines is eminent in the central part of the spectra (Figure 1). Above the coalescence temperature, the 
spectra can be interpreted as a triplet of triplet respectively due to the two equivalent phosphorous couplings and the nitrogen. The nine major lines have a further small doublet splitting due to the proton coupling. The central lines of the 1:2:1 phosphorus triplet always have reduced amplitudes indicating the presence of a chemical exchange. Such a behavior is expected when the N-C rotation is restricted and the exchange takes place between two mirror conformers. However, this model cannot explain the spectra recorded below the coalescence temperature: seven lines (Figure 1, 183 pentane) appear in the center of the spectra whereas the symmetric model can only produce a maximum of six lines.

a)

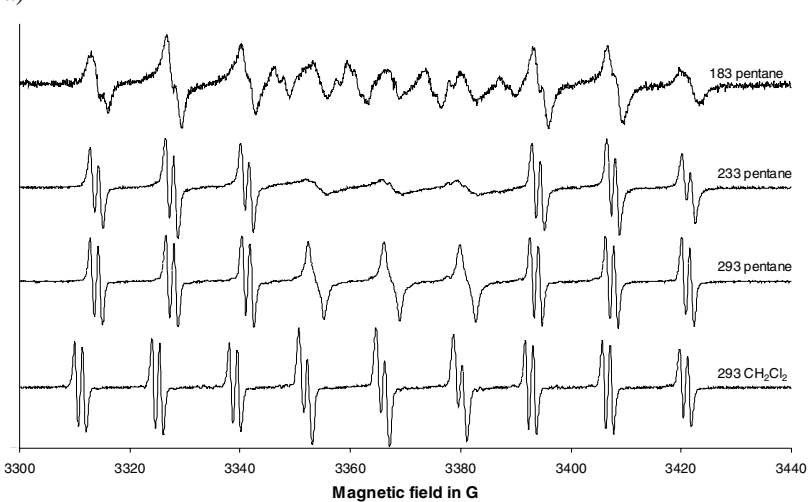

b)

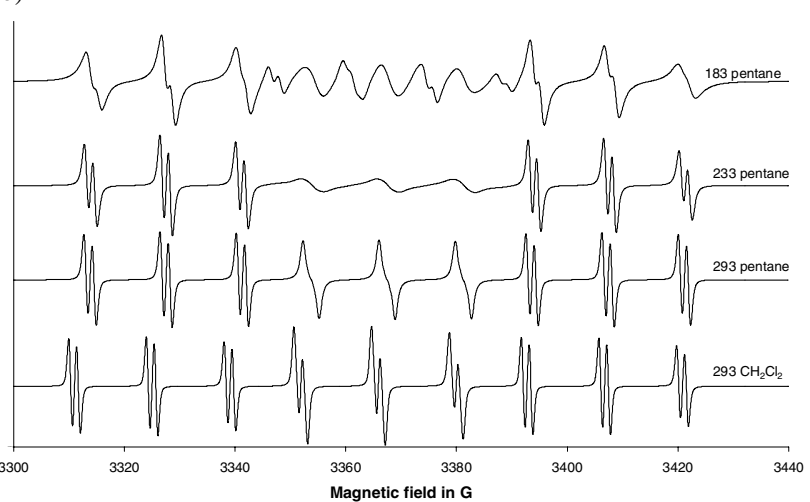

Figure 1. a) Experimental and b) simulated ESR spectra of radical $\mathbf{1}$ in different solvents at several temperatures.

In order to resolve this contradiction, we applied an asymmetric two-site model in which the conformers are not mirror images. ${ }^{10}$ It offered an excellent fit for all the temperatures. Above the coalescence region, this model gave a much larger statistical error in the quality of the fit when compared to the symmetric model which also provides a qualitatively good simulation. In the low temperature region, the computations suggested that the two phosphorus atoms are closely equivalent for one of the conformers (both phosphorous couplings are $40 \mathrm{G}$ in pentane whereas the couplings are $41 \mathrm{G}$ and $43 \mathrm{G}$ in dichloromethane). For the other conformer, the two $\mathrm{A}_{\mathrm{p}}$ couplings are different, 32.5 $\mathrm{G}$ and $47.5 \mathrm{G}$ in pentane, while being $35.5 \mathrm{G}$ and $46.5 \mathrm{G}$ in dichloromethane. The quality of the fit was 
significantly improved below the coalescence region if the proton couplings were also different: the hydrogen splitting was always smaller for the symmetric conformer (Figure 2).

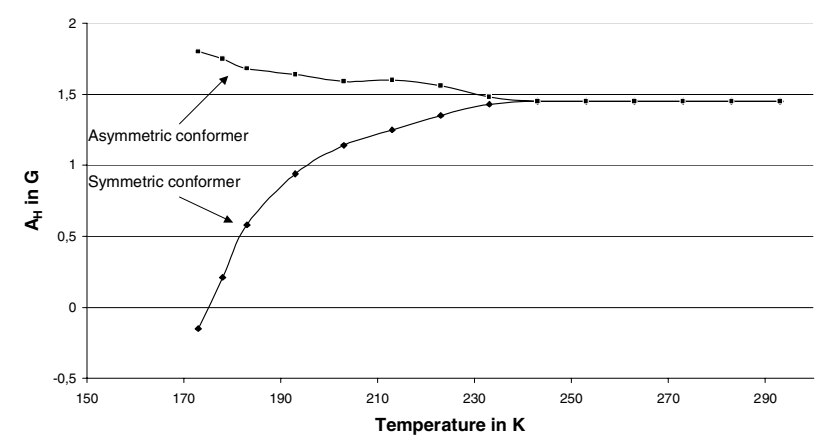

Figure 2. Variation of $A_{H}$ for the two conformers of radical 1 in pentane.

Interestingly, the relaxation parameters describing the intrinsic line width (i.e. without exchange broadening) were significantly larger for the symmetric conformer in pentane (Figure 3) whereas they only slightly differed in dichloromethane.

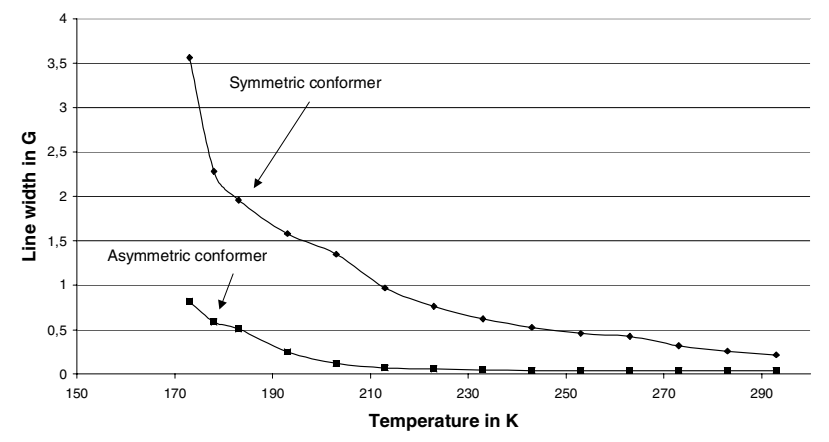

Figure 3. Line width of radical 1 in pentane.

All the above observations can be explained by the hindered rotation of the phosphoryl groups around the C-P bonds. The small value of the proton coupling shows that the rotation around the N-C 
bond is completely blocked and that the dihedral angle between the $\mathrm{NCH}$ and $\mathrm{CNp}_{z}$ planes is perpendicular ( $\mathrm{p}_{\mathrm{z}}$ describes the lobe of an unpaired electron). The phosphoryl group can be seen as an asymmetric rotor. Considering that the rotation around the C-P bond has three non-eclipsing positions, a set of nine conformers arises from the presence of the two phosphoryl groups. ${ }^{11} \mathrm{P}_{\alpha}, \mathrm{P}_{\beta}$ and $\mathrm{P}_{\beta}{ }^{\prime}$ refer to the three phosphoryl orientations where $\mathrm{P}_{\alpha}$ is symmetric on the NCP plane whereas $\mathrm{P}_{\beta}$ and $\mathrm{P}_{\beta}^{\prime}$ are mirror images (Figure 4). Three of the conformers pairs have a mirror symmetry with respect to the $\mathrm{CNp}_{\mathrm{z}}$ plane whereas the other six don't.

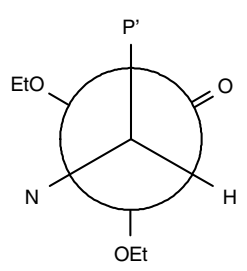

$P_{\alpha}$

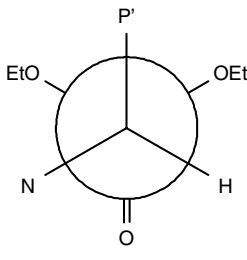

$P_{\beta}$

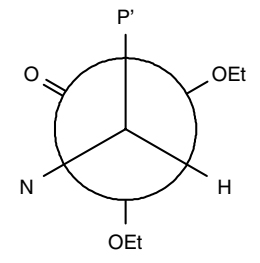

$P_{\beta^{\prime}}$

Figure 4. CP' eclipsing Newman diagrams showing the $\mathrm{P}_{\alpha}$ symmetric orientation of the $\mathrm{P}^{\prime}(\mathrm{O})(\mathrm{OEt})_{2}$ group to the NCP' plane and showing when the conformations form mirror images $\mathrm{P}_{\beta}$ and $\mathrm{P}_{\beta}$ '.

However, the number of conformations is much larger if the internal rotations of the ethoxy groups around the P-O, O-C and C-C sigma bonds are considered. Between the possible conformers, two major groups can be distinguished according to their symmetry properties with respect to the $\mathrm{CNp}_{z}$ plane, namely the symmetric and asymmetric conformers. In the first group, the two phosphorus atoms are equivalent and the $\mathrm{NCH}$ plane should be perfectly perpendicular to the $\mathrm{CNp}_{z}$ plane. Select $\left(\mathrm{P}_{\alpha,} \mathrm{P}_{\alpha}\right)$ and neglect $\left(\mathrm{P}_{\beta}, \mathrm{P}_{\beta}\right)$ as energetically unfavorable from the symmetric pairs and denote by $\left(\mathrm{P}_{\alpha}\right.$, $\left.\mathrm{P}_{\beta}\right)$ and $\left(\mathrm{P}_{\beta}, \mathrm{P}_{\alpha}\right)$ the thermally accessible asymmetric geometry. If the C-P rotations are independent,

$$
\left(\mathrm{P}_{\alpha}, \mathrm{P}_{\alpha}\right) \leftrightarrow\left(\mathrm{P}_{\alpha}, \mathrm{P}_{\beta}\right) \text { and }\left(\mathrm{P}_{\alpha}, \mathrm{P}_{\alpha}\right) \leftrightarrow\left(\mathrm{P}_{\beta}, \mathrm{P}_{\alpha}\right)
$$


transitions can occur, while the concerted C-P rotations can yield to direct processes:

$$
\left(\mathrm{P}_{\alpha}, \mathrm{P}_{\beta}\right) \leftrightarrow\left(\mathrm{P}_{\beta}, \mathrm{P}_{\alpha}\right)
$$

If the concerted rotations are slow, the asymmetric two-site exchange processes in (1) will represent the predominant broadening mechanism whereas the symmetric (2) processes should be considered in the fast case. In the intermediate rate, only a four-site exchange model could offer an adequate description. As the asymmetric two-site exchange model gave an excellent spectrum fit on the entire temperature range, it is sufficient to consider the independent $\mathrm{C}-\mathrm{P}$ rotations of (1) only.

The site with closely identical $A_{P}$ couplings can be assigned to $\left(\mathrm{P}_{\alpha,} \mathrm{P}_{\alpha}\right)$, and the other one with different couplings to $\left(\mathrm{P}_{\alpha}, \mathrm{P}_{\beta}\right)$. Whereas the proton coupling tends to zero or a small negative value for the former site in the low temperature region, it can have a slightly larger value for the asymmetric site where the phosphorus atoms are not equivalent. This reveals that the NCH plane is not perpendicular to the $\mathrm{CNp}_{z}$ plane anymore. Since a lot of conformers amount to closely identical energies, we couldn't expect that only a single geometry is thermally populated and, therefore, a spread of geometries should be taken into account. Consequently, the symmetry of $\left(\mathrm{P}_{\alpha}, \mathrm{P}_{\alpha}\right)$ is imperfect and this explains why the two $A_{P}$ couplings are slightly different in dichloromethane. Furthermore, the dependence of the relative populations on temperature shows a rather complex behavior: in dichloromethane, two linear regions can be distinguished on the Arrhenius plot (Figure 5).

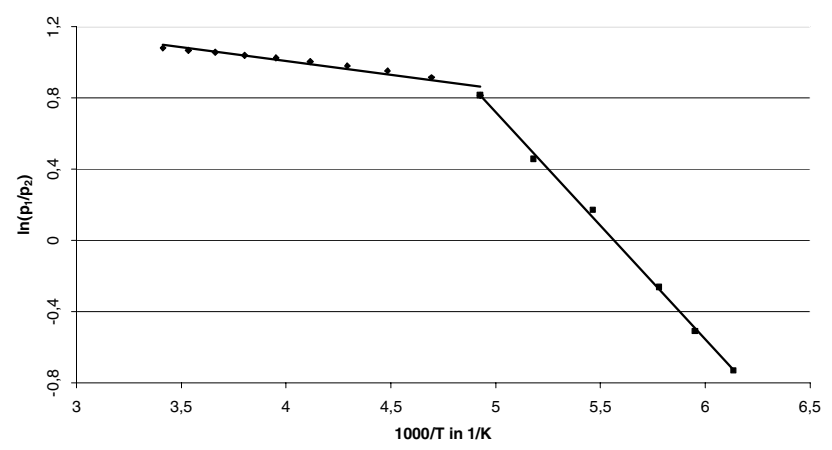


Figure 5. Relative populations of symmetric versus asymmetric sites for $\mathbf{1}$ in dichloromethane.

In pentane, the strongly different line width makes the estimate of the relative populations rather uncertain but similar trends can be expected. At low temperature, the increasing population of an asymmetric conformer suggests its preference. The slope gives an energy separation of ca. $10 \mathrm{~kJ} / \mathrm{mol}$ (Figure 5). This value is reduced by an order of magnitude at higher temperature where the thermal accessibility of further conformers can strongly alter the relative population of the two groups. The drastic solvent effect on the exchange time (Figure 6) and on the relaxation parameters (Figure 3) can be explained by molecular associations between the solvent molecules and the nitroxide radicals. It is assumed that the chelating phosphoryl groups can 'trap' the pentane molecule in the symmetric conformation. These molecular associations can hinder the complex motion of the phosphoryl groups as well as it enhances the hydrodynamic radius of the molecule. As a consequence, the exchange jumps and molecular reorientations should slow down. If the lifetime of the solvent-solute adduct is long in the chelating form and short in the other configuration, the exchange rate is then unaffected by the kinetics of the solvent-solute associations. This assumption is sustained by the fact that the same potential barrier is obtained $(14 \mathrm{~kJ} / \mathrm{mol})$ from the Arrhenius plot of the average exchange time in the two solvents (Figure 6). The dichloromethane polarity only plays an important role when the solventsolute association takes place at the polar NO moiety which manifests itself by the increased value of the Bp coefficient.

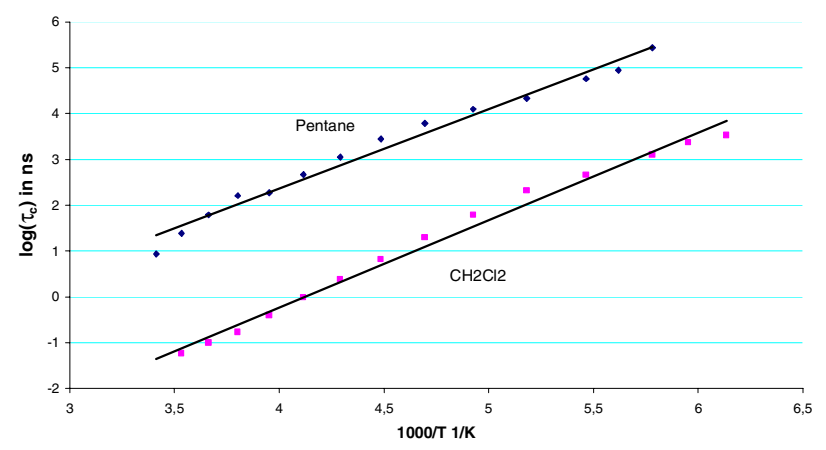


Figure 6. Arrhenius plot of exchange time for 1.

The $\vartheta^{\prime}$ and $\vartheta "$ dihedral angles (Figure 7) defined for the phosphorus atoms can be related to the $\Theta$ dihedral angle between the NCP' and NCP" planes:

$$
\vartheta^{\prime}+\vartheta^{\prime \prime}=180-\Theta
$$

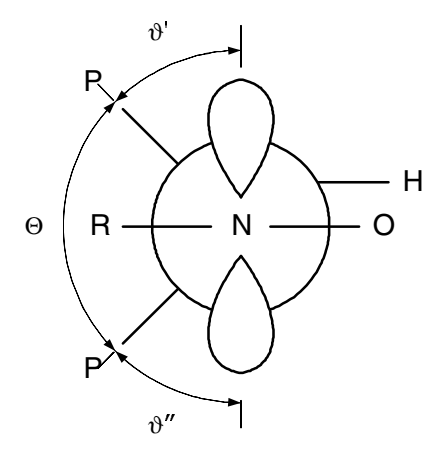

Figure 7. NC eclipsing Newman diagram showing the $\vartheta^{\prime}, \vartheta^{\prime \prime}$ and $\Theta$ dihedral angles as defined

By assuming $\Theta=120^{\circ}$, the dihedral angles for the equivalent phosphorus amount to $30^{\circ}$, which gives the coefficient $\mathrm{B}_{\mathrm{P}}=53.3 \mathrm{G}$ in the usual dihedral relation ${ }^{12,13}$

$$
A_{P}=B_{P} \cos ^{2} \vartheta
$$

when $A_{p}^{\prime}=A_{p}^{\prime \prime}=40 \mathrm{G}$ for the symmetric site in pentane. The larger $A_{p}$ splitting (42 $\left.G\right)$ in dichloromethane yields an enhanced $B_{P}=56 \mathrm{G}$ value. The sum of $A_{P}$ couplings from Eqs (3) and (4) is given by:

$$
A_{P^{\prime}}+A_{P^{\prime \prime}}=2 B_{P}\left(\cos ^{2} \frac{\Theta}{2}-\cos \Theta \cos ^{2} \frac{\vartheta^{\prime}-\vartheta^{\prime \prime}}{2}\right)
$$


Here $\cos ^{2}\left(\left(\vartheta^{\prime}-\vartheta^{\prime \prime}\right) / 2\right)$ has a maximum when $\vartheta^{\prime}=\vartheta^{\prime \prime}$; since $\cos \Theta$ is negative, Eq (5) suggests a reduction of the sum of the couplings for the asymmetric site, in contrast to the observation that, in pentane, $\mathrm{A}_{\mathrm{p}}{ }^{\prime}+\mathrm{A}_{\mathrm{p}}{ }^{\prime \prime}$ is $80 \mathrm{G}$ for both conformers. It means that the reorientation of the phosphoryl groups also produces a change in the $\mathrm{CP}_{2}$ bonding angle. In pentane, the actual couplings $\mathrm{A}_{\mathrm{p}}{ }^{\prime}=47.5 \mathrm{G}$ and $\mathrm{A}_{\mathrm{p}}{ }^{\prime \prime}=32.5 \mathrm{G}$ give the corresponding dihedral angles $\vartheta^{\prime}=19.3^{\circ}$ and $\vartheta^{\prime \prime}=38.7^{\circ}$; their sum amounts to $58^{\circ}$ and is lower, by two degrees, than the value of $\vartheta^{\prime}+\vartheta^{\prime \prime}=60^{\circ}$ assumed for the symmetric conformer where the $\vartheta^{\prime}=\vartheta^{\prime \prime}=30^{\circ}$ angle can reproduce the experimental couplings. Naturally, we arbitrarily used $\vartheta^{\prime}=\vartheta^{\prime \prime}=30^{\circ}$ when the $\mathrm{B}_{\mathrm{P}}=53.3 \mathrm{G}$ value was derived but this choice doesn't affect our statement that the angle should be larger by approximately two degrees for the asymmetric conformer. The small angular deformation between the symmetric and non-symmetric conformers can accidentally offset the reduction of the sum of the phosphorus couplings due to the non-symmetric orientation of the phosphoryl groups. This compensation is more accurate in pentane, where the solvent molecule is associated with the chelating phosphoryl groups, than in dichloromethane, where the distortion of the $\mathrm{CP}_{2}$ bonding angle seems to be smaller. In the latter case, the sum of couplings is slightly different for the two sites: $84 \mathrm{G}$ for symmetric and $82 \mathrm{G}$ for the asymmetric conformer.

\section{Analysis of Radical 2}

The ESR spectra of $\mathbf{2}$ in toluene consist of the hyperfine splitting due to two phosphori, two protons and one nitrogen (Figure 8). At higher temperature, a triplet of $9.3 \mathrm{G}$ can be seen in the tails of the spectra ; it reveals a fast reorientation of the $\mathrm{CH}_{2}$ group. The central pattern of the spectra can be interpreted by assuming two different $\mathrm{A}_{\mathrm{P}}$ couplings of $31.5 \mathrm{G}$ and $38 \mathrm{G}$, i.e. no $\mathrm{CP}_{2}$ rotation exists. Below $273 \mathrm{~K}$, the rotation around $\mathrm{N}-\mathrm{C}$ bond is also blocked, yielding two small proton couplings of 5.5 
$\mathrm{G}$ and $8 \mathrm{G}$. At all temperatures, a fairly good fit is obtained by using a two-site exchange model. Representative couplings at two different temperatures are described in Table 2.

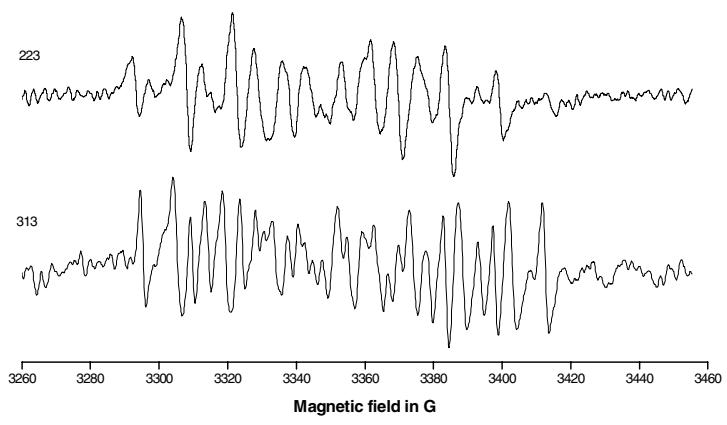

Figure 8. Experimental ESR spectra of radical 2 in toluene at 223 and $313 \mathrm{~K}$.

Table 2: Hyperfine couplings in the two-site exchange model of radical 2.

\begin{tabular}{|c|c|c|c|c|}
\hline \multirow{2}{*}{$\begin{array}{l}\text { Temperature } \\
\text { /K } \\
\text { Coupling /G }\end{array}$} & \multicolumn{2}{|c|}{313} & \multicolumn{2}{|c|}{223} \\
\hline & Site A & Site B & Site A & Site B \\
\hline$A_{N}$ & 14.6 & 14.5 & 14.3 & 14.8 \\
\hline $\mathrm{A}_{\mathrm{p}}{ }^{\prime}$ & 32.2 & 20.0 & 28.6 & 31.3 \\
\hline $\mathrm{A}_{\mathrm{p}} "$ & 36.5 & 49.5 & 39.6 & 40.2 \\
\hline $\mathrm{A}_{\mathrm{H}}{ }^{\prime}$ & 11.6 & 7.5 & 14.2 & 5.5 \\
\hline $\mathrm{A}_{\mathrm{H}}{ }^{\prime \prime}$ & 9.1 & 10.2 & 7.9 & 15.7 \\
\hline
\end{tabular}

The description of the conformational motions would require at least a four-site exchange model, ${ }^{14}$ since both the previously discussed C-P rotations between the symmetric and asymmetric phosphoryl positions and the rotation around the $\mathrm{N}-\mathrm{C}$ bond of the $\mathrm{CH}_{2} \mathrm{Ph}$ group should be considered. The two-site model could only offer effective couplings and, due to the relative populations change in the four-site model, the calculated couplings show a significant dependence on temperature. Table 2 shows that the phosphorous couplings are close in site A and strongly different in site B at higher temperature. In 
these conditions, the $\mathrm{CH}_{2} \mathrm{Ph}$ rotation is fast and its impact on the motion of the phosphoryl groups is thus similar to the effect of a tertio-butyl group in radical 1. At lower temperature where the rotation of the $\mathrm{CH}_{2} \mathrm{Ph}$ group is frozen, the symmetry is broken with respect to the $\mathrm{CNp}_{z}$ plane and its impact on the orientation of the C-P bonds will be more important than the mutual position of the phosphoryl rotamers. This explains why the values of $\mathrm{A}_{\mathrm{P}}{ }^{\prime}$ and $\mathrm{A}_{\mathrm{P}}{ }^{\prime \prime}$ are closely identical for site $\mathrm{A}$ and $\mathrm{B}$.

\section{Analysis of Radical 3}

Above room temperature, nine major lines appear in the ESR spectra due to the primary phosphorus and the secondary nitrogen triplets (Figure 9). The central lines of the phosphorus triplet have smaller amplitudes than the outer lines, revealing a strong chemical exchange. At high temperature (363 K in toluene) all the lines display a further tertiary structure ; the three center lines produce a small 1:2:1 triplet pattern whereas each line of the outer triplets splits into a small doublet pattern. This splitting can be attributed to the beta proton of the sec-butyl group in an orientation where the $\mathrm{NCH}$ plane is closely perpendicular to the $\mathrm{CNp}_{z}$ plane. The tertier triplet pattern of the central nitrogen lines can be explained by the chirality of R": even in the case of a fast rotation of the phosphoryl groups, the two phosphori remain non-equivalent and the difference of $\mathrm{A}_{\mathrm{P}}$ couplings accidentally agrees with the small proton coupling.

a)

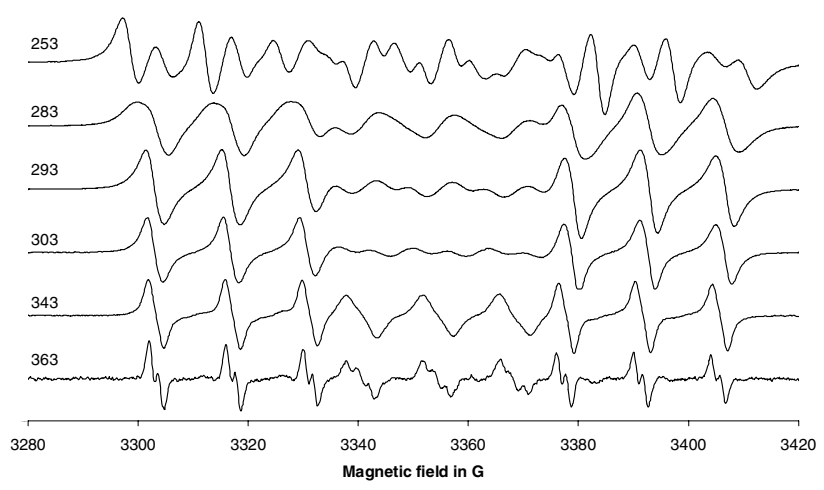

b)

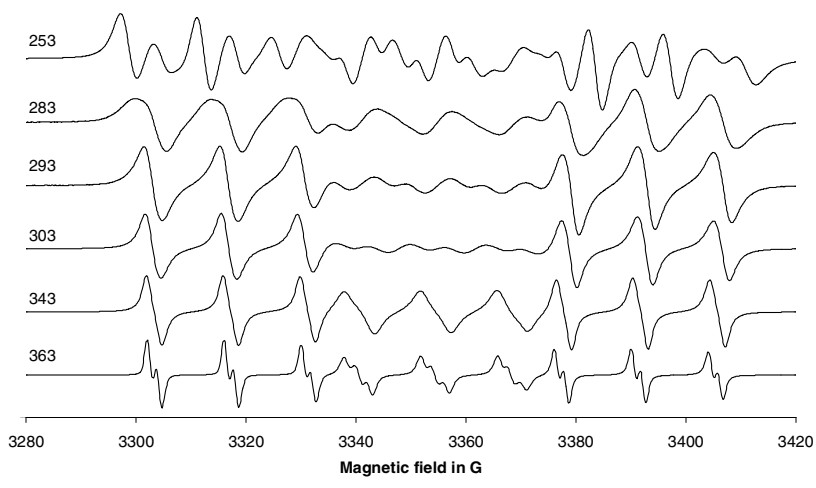


Figure 9. a) Experimental and b) simulated ESR spectra of radical 3 in toluene at several temperatures.

By lowering the temperature from $363 \mathrm{~K}$, the lines start to broaden and only the nine major lines without further splitting can be observed. In toluene below $333 \mathrm{~K}$ (Figure 9) and in dichloromethane below $343 \mathrm{~K}$, a new doublet splitting appears in the three center lines and the relative amplitudes of the inner lines are gradually decreasing with temperature. The spectra change dramatically at $293 \mathrm{~K}$ in toluene and at $283 \mathrm{~K}$ in dichloromethane: the position of the lines in the central part is completely reorganized. This reveals the transition from a fast exchange to a slow exchange condition. Further decreasing the temperature by ten degrees leads to an additional broadening of both the outer and the central lines. As a consequence, only four lines remain in the center. This broadening stops around 280 $\mathrm{K}$ and new structures appear both in the center and in the wings. The outer lines split into an asymmetric doublet, while the two center lines produce a triplet ; 20 - 22 separate lines can altogether be distinguished. By decreasing the temperature even further, the structure becomes more and more irregular while, at $213 \mathrm{~K}$ in toluene and at $203 \mathrm{~K}$ in dichloromethane, the characteristic peaks of rigid spectra appear. All the above spectral changes were found reversible unless the temperature is increased up to $363 \mathrm{~K}$, when a partial decomposition of the radical and the formation of new species can be observed.

The complexity of the spectrum variations reveals that the exchange mechanism is highly complex and has at least two coalescence regions. Above the first coalescence, a good simulation can be achieved by using an asymmetric exchange model. In this region, it is assumed that the rotation of the phosphoryl groups is fast and the exchange broadening is primarily caused by the hindrance of the R" rotation around the $\mathrm{C}-\mathrm{N}$ bond. The Arrhenius plot gives a $35 \mathrm{~kJ} / \mathrm{mol}$ potential barrier for this motion (Figure 10). When this rotation is slowing down, the phosphoryl rotation also contributes to the LWA. Consequently, a second coalescence appears in the center of the spectra and the exchange takes place at least via four different sites. By assuming a model in which two independent asymmetric exchange 
processes are superimposed, an excellent fit for the spectra is obtained on the entire temperature range. Since this model works with 16 hyperfine couplings (one nitrogen, two phosphori and one proton for each site) there is a possibility that a good fit can be achieved by alternative parameter sets. For this reason, only tentative conclusions can be drawn from the results of the spectrum simulations. Let us denote by $\mathrm{A}^{\prime}$ and $\mathrm{B}^{\prime}$ the sites of the exchange process that is produced primarily by the $\mathrm{R}$ " rotation around the $\mathrm{C}-\mathrm{N}$ bond. In $\mathrm{A}^{\prime}$, the proton coupling is small (less than $1 \mathrm{G}$ ) i.e. the $\mathrm{CH}$ bond is eclipsing with NO. In this site, the two phosphorus atoms have slightly different couplings: respectively 40 and $45 \mathrm{G}$. In site B', a larger proton splitting $(6-10 \mathrm{G})$ is observed. It produces the doublet structure that we noticed in the wings below the coalescence region. The dihedral angle between the $\mathrm{NCH}$ and $\mathrm{CNp}_{z}$ planes can be estimated at 50 - 60 degrees. It is in accordance with the strongly different phosphorus couplings of 25 and $50 \mathrm{G}$. One phosphorus has a small and the other one has a rather large dihedral angle with the plane of the unpaired electron. The second exchange process with sites A" and B" can be ascribed to the internal rotations of the phosphoryl groups. Here A" has a larger (max $5 \mathrm{G})$ and $\mathrm{B}$ " a small or negative proton coupling. As concerning the phosphorus couplings, they are strongly different for A" (45 and $20 \mathrm{G}$ ) and the difference is smaller for B" (31 and $43 \mathrm{G})$. Presumably A" corresponds to the $\left(\mathrm{P}_{\alpha}, \mathrm{P}_{\beta}\right)$ and $\left(\mathrm{P}_{\beta}, \mathrm{P}_{\alpha}\right)$ sites. Due to the chirality, the sites $\left(\mathrm{P}_{\alpha,} \mathrm{P}_{\beta}\right)$ and $\left(\mathrm{P}_{\beta}, \mathrm{P}_{\alpha}\right)$ should be different. $\mathrm{B}$ " can be assigned to a $\left(\mathrm{P}_{\alpha}, \mathrm{P}_{\alpha}\right)$ state. Here, the difference of $\mathrm{A}_{\mathrm{P}}$ couplings can be explained by the chirality of the molecule. ${ }^{3}$ Since the decomposition of the complex exchange phenomenon to simpler two-site processes only gives effective sites, both the phosphorus and proton couplings reveal a significant temperature dependence. 


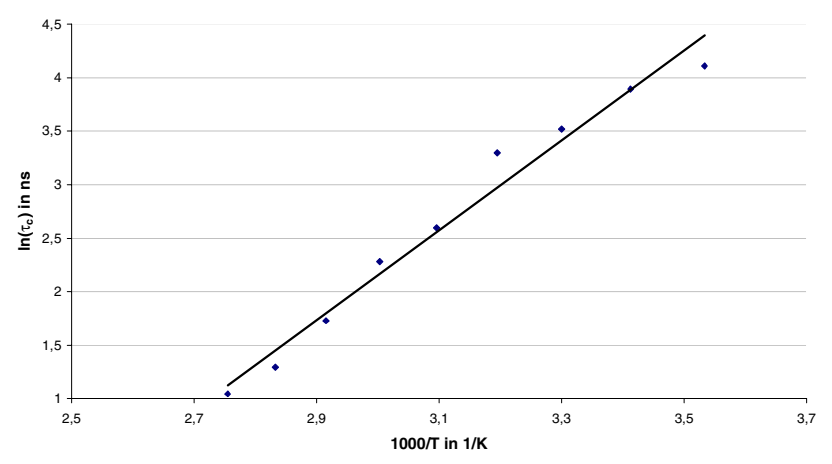

Figure 10. Arrhenius plot of exchange time for 3 .

\section{Conclusions}

The ESR spectra of geminally diphosphorylated linear nitroxide radicals present LWA regardless of the presence or the absence of a chiral center. The analysis of the line structure recorded in the slow motional case reveals the pseudo-chiral character of the geminally substituted carbon atoms. This pseudo-chirality is produced by the different local geometries of the phosphoryl groups, when the steric repulsion between the geminal substituents can be minimized if the steric arrangements around the six flexible $\sigma$-bonds are not identical. If there is no other chiral center in the molecule, the LWA can be described by the chemical exchange between a symmetric and an asymmetric conformer. This motion also affects the bonding angle in the $\mathrm{CP}_{2}$ group. It is reduced by 2 degrees when the phosphori become non-equivalent. In pentane, the rate of exchange is significantly lowered while the magnetic relaxation is intensified. This can be explained by the trapping of a solvent molecule between the chelating phosphoryl groups. If the $\mathrm{NO}$ moiety is linked to a $\mathrm{CH}_{2}$ group or to a chiral center, the exchange phenomena produce two coalescences which can be described by a four-site exchange model.

\section{Acknowledgements}


We thank the Hungarian Scientific Research Fund OTKA T-032929 for the financial support.

\section{References and Notes}

(1) Rockenbauer, A.; Mercier, A.; Le Moigne, F.; Olive, G.; Tordo, P. J. Phys. Chem. A 1997, 101 (43), 7965.

(2) ${ }^{a}$ Gilbert, B. C.; Larkin J. P.; Norman R. O. C. J. Chem. Soc., Perkin Trans. 2 1972, 1272. ${ }^{\mathrm{b}}$ Franchi, P.; Lucarini, M.; Pedulli, G. F.; Bandini, E. Chem. Comm. 2002, 560.

(3) Hashimoto, K.; Togo, H.; Morihashi, K.; Yokoyama, Y.; Kikuchi, O. Bull. Chem. Soc. Jpn. 1991, 64, 3245 .

(4) Olive, G.; Le Moigne, F.; Mercier, A.; Tordo, P. Synth. Commun. 2000, 30 (4), 619.

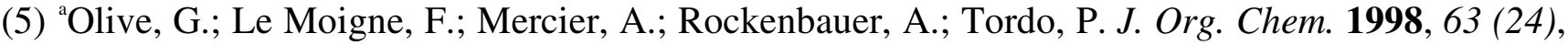
9095. 'Olive, G.; Jacques, A. Phosphorus, Sulfur, Silicon Relat. Elem., 2003, 178 (1), 33.

(6) Levin, Y. A.; Il'yasov, A. V.; Mukhtarov, A. S.; Skorobogotova, M. S. Teor. Eksper. Khim. 1975, $11(5), 612$.

(7) Alberti, A.; Hudson, A.; Pedulli, G. F. Tetrahedron 1984, 40 (23), 4955.

(8) Rockenbauer, A.; Korecz, L. Appl. Magn. Reson. 1996, 10, 29.

(9) Lock, M. V.; Sagar, B. F. J. Chem. Soc. (B) 1966, 690

(10) Stevenson, C. D.; Kim, Y. S. J. Am. Chem. Soc. 2000, 122, 3211.

(11) Belostotskii, A. M.; Gottlieb, H. E.; Aped, P.; Hassner, A. Chem.-Eur. J. 1999, 5, 449.

(12) McConnell, H. M. J. Chem. Phys. 1956, 24, 632.

(13) Heller, C.; McConnell, H. M. J. Chem. Phys. 1960, 32 (5), 1535. 
(14) ${ }^{\mathrm{a}}$ Bolton, J. R.; Carrington, A. Mol. Phys. 1962, 5, 161. ${ }^{\mathrm{b}}$ Carrington, A. Mol. Phys. 1962, 5,425. ${ }^{\mathrm{c}}$ Bolton, J. R.; Carrington, A; Todd, P. F. Pol. Phys. 1963, 6, 169. ${ }^{\mathrm{d}}$ Yamazaki, I.; Piette, L. H. J. Am. Chem. Soc. 1965, 87, 986. 'Smith, I. C. P.; Carrington, A. Mol. Phys. 1967, 12, 439. ${ }^{\text {f Loth, }}$ K.; Graf, F.; Günthard, Hs. H. Chem. Phys. 1976, 13, 95. 